\title{
Cluny, le monachisme et la société au premier âge féodal (880-1050)
}

Romainmôtier, 24-26 juin 2010 et Cluny, 9-11 septembre 2010

Dominique logna-Prat

\section{(2) OpenEdition}

\section{Journals}

Édition électronique

URL : https://journals.openedition.org/cem/11954

DOI : 10.4000/cem. 11954

ISSN : 1954-3093

Éditeur

Centre d'études médiévales Saint-Germain d'Auxerre

Édition imprimée

Pagination : 139-168

ISSN : 1623-5770

Référence électronique

Dominique logna-Prat, «Cluny, le monachisme et la société au premier âge féodal (880-1050)». Bulletin du centre d'études médiévales d'Auxerre / BUCEMA [En ligne], 15 | 2011, mis en ligne le 14 décembre 2011, consulté le 22 septembre 2022. URL : http://journals.openedition.org/cem/11954 ; DOI : https://doi.org/10.4000/cem.11954

Ce document a été généré automatiquement le 22 septembre 2022.

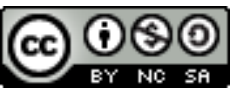

Creative Commons - Attribution - Pas d'Utilisation Commerciale - Partage dans les Mêmes Conditions 4.0 International - CC BY-NC-SA 4.0

https://creativecommons.org/licenses/by-nc-sa/4.0/ 


\section{Cluny, le monachisme et la société au premier âge féodal (880-1050)}

Romainmôtier, 24-26 juin 2010 et Cluny, 9-11 septembre 2010

Dominique logna-Prat

1 À l'occasion du XI centenaire de la fondation de Cluny (910-2010), ce colloque international en deux volets invitait à discuter les apports historiographiques les plus récents et les recherches en cours sur les origines et les premiers temps de Cluny, mais en dépassant largement le seul horizon du monastère bourguignon - rôle des moines dans la confusion Église/société; importance des usages monastiques dans la conception de l'échange; inscription spatiale des pouvoirs; fonctionnalité des pratiques artistiques et monumentales... Le but assigné par les organisateurs était d'étudier le monachisme comme agent de transformation sociale en un temps charnière, celui de la constitution d'une société seigneuriale caractérisée par les rapports de domination: domination des hommes, domination de la terre et contrôle des échanges. En quoi, les seigneuries ecclésiastiques comme Cluny ont-elles pu participer de ce mouvement général, constitutif du « féodalisme »? Dans quelle mesure la « sacralité » des terres de saint Pierre a-t-elle conféré à cette seigneurie, cœur d'une véritable "Église», un statut spécifique? Telles étaient les principales questions d'histoire sociale que ce colloque entendait poser, en recourant à toutes les approches possibles (culturelles, politiques, économiques), au point de rencontre entre les diverses spécialités académiques intéressées par l'histoire de Cluny : histoire, histoire de l'art, archéologie, liturgie et musicologie.

\section{Premier volet : Romainmôtier, 24-26 juin 2010}

2 Le premier volet du colloque était intitulé : « Cluny, le monachisme et l'Église ». Dans la dynamique d'ensemble des deux rencontres, Barbara H. Rosenwein a, dans sa leçon inaugurale, commencé par poser la question des rapports entre « Réforme monastique et transformation sociale ». Les cinq premières sections du colloque devaient permettre de fournir une vue comparée du premier Cluny, replacé aussi bien dans son contexte 
social (le monde seigneurial) que dans la logique des réformes monastiques et canoniales tardo-carolingiennes, cela dans toutes les formes d'expression du "phénomène » clunisien auquel la documentation nous donne accès - sources écrites, visuelles et monumentales. Le cadre comparatif a permis, dans une sixième section, de s'intéresser à Cluny dans l'horizon de la Bourgogne transjurane - coordination, JeanDaniel Morerod, contributions de Gilbert Coutaz et Alexandre Pahud.

3 La première matinée du colloque était organisée en deux volets - coordonnés par Isabelle Rosé. L'objectif était d'appréhender les pratiques sociales et les idéologies monastiques en regard de celles de ces autres acteurs ecclésiastiques que sont les chanoines et les évêques, au cours du $\mathrm{x}^{\mathrm{e}}$ siècle, moment de lente émergence du premier âge féodal. Comment cerner ce qui fait l'unité et la pluralité de l'Église, en tant que structure sociale, étant entendu que les parcours de vie sont extrêmement divers au cours de cette période et qu'ils sont souvent caractérisés par le passage fréquent d'un modèle de régularité à un autre - de chanoine à moine, avec de nombreuses expériences érémitiques? La question revenait à replacer le Cluny des origines dans son contexte ecclésial, à la fois au sein du monde monastique en pleine évolution tout au long $\mathrm{du} \mathrm{x}^{\mathrm{e}}$ siècle, et face au monde de l'Église séculière avec lequel il a été tantôt en concurrence, tantôt en collaboration étroite.

\section{Le monachisme, un agent de transformation pour la société et pour l'Église}

4 Le premier volet envisageait le monachisme, qu'il soit incarné par Cluny ou par les congrégations monastiques, comme révélateur et laboratoire d'une société nouvelle, à la fois dans ses pratiques sociales et dans sa manière de penser et de théoriser l'émergence de formes de pouvoir inédites.

5 La communication d'Isabelle Rosé, «Le premier Cluny », a été consacrée au monastère de Cluny dans la première moitié $\mathrm{du} \mathrm{x}^{\mathrm{e}}$ siècle, depuis sa fondation par le couple princier formé par le duc d'Aquitaine, Guillaume le Pieux, et son épouse bosonide Engelberge, jusqu'au tournant marqué par les débuts de l'abbatiat d'Aymard. Cette période de l'histoire de l'abbaye, longtemps appréhendée dans une perspective téléologique - par la recherche des ferments des succès futurs de l'Ecclesia puis de l'Ordo clunisiens -, a été revisitée par des études importantes à partir des années 1980-1990 - B. H. Rosenwein, D. Iogna-Prat, G. Constable et D. Méhu -, qui en ont souligné les enjeux propres. Il s'agissait cette fois de mettre en perspective les premiers temps de Cluny, sur les plans tout à la fois matériel, social et idéologique, à une période de bouleversements sociaux et politiques importants dans le monde aristocratique, avec un effacement progressif des structures carolingiennes et la genèse d'une société seigneuriale. Dans cette perspective, le premier Cluny, en tant que microcosme peuplé d'aristocrates extrêmement bien insérés dans la noblesse du siècle, qui sont de surcroît des hommes de plume, apparaît comme un révélateur de cette période de transition, qui voit l'émergence de nouvelles formes sociales.

6 Le premier Cluny permet tout d'abord de mettre en lumière les relations complexes entretenues par le monde monastique avec les pouvoirs des princes séculiers ou ceux des cadres ecclésiastiques que sont les évêques. À partir des exemples de Bernon et d'Odon, il est possible de suivre les stratégies réticulaires, développées par les premiers abbés de Cluny, pour entretenir des relations avec l'ensemble du monde aristocratique. 
D'un intérêt tout spécial : le développement de pratiques de multi-appartenance à plusieurs milieux nobiliaires, qui permettent d'asseoir le pouvoir des acteurs monastiques.

7 Dans un deuxième temps, Isabelle Rosé s'est efforcée d'éclairer l'émergence de ce pouvoir monastique d'un nouveau type, incarné par des multi-abbatiats à large échelle, qui résultent d'une diffusion de l'idéal réformateur tel qu'il avait été pensé par Benoît d'Aniane un siècle auparavant. Elle s'est interrogée sur les modalités de construction de ces ensembles, qui reposent sur l'insertion des réformateurs dans les réseaux aristocratiques, sur leur grande mobilité géographique et sur la gestion du pouvoir dans chaque abbaye avec un co-abbé. À ce titre, les multi-abbés du premier Cluny apparaissent comme les derniers héritiers d'un certain idéal carolingien, où l'exercice du pouvoir était lié à l'itinérance de ses titulaires à l'échelle de l'Empire, mais aussi comme des proto-seigneurs dont l'autorité est disséminée et partagée avec d'autres à l'échelle locale.

8 Le premier Cluny constitue enfin un bon révélateur des modifications qui affectent la société $\mathrm{du} \mathrm{x}^{\mathrm{e}}$ siècle dans la mesure où son deuxième abbé, Odon, a laissé une œuvre d'une richesse exceptionnelle pour l'époque, qui permet d'appréhender la manière dont le monde monastique a décrit, théorisé et légitimé la genèse d'un monde nouveau. Il en ressort une vision sociale binaire originale, qui privilégie les relations, sous forme d'aumônes, entre de puissants laïcs, qui exercent la force légitime et mettent leur glaive au service de l'Église, et des moines réformés et purs, qui s'identifient à des pauvres symboliques. Un tel schéma, en rupture avec les théories de l'époque carolingienne, légitime la position sociale nouvelle des princes et surtout des moines, en plaçant ces derniers en position de domination.

9 Noëlle Deflou-Leca, «Réforme et réseaux de dépendances dans le monachisme postcarolingien ( $\mathrm{x}^{\mathrm{e}}-\mathrm{XI}^{\mathrm{e}}$ siècle)», s'est ensuite intéressée à l'essor des réseaux de dépendances monastiques dans le contexte réformateur des $\mathrm{x}^{\mathrm{e}}$ et $\mathrm{xI}^{\mathrm{e}}$ siècles, suivant deux axes de réflexion, en adoptant, dans un premier temps, une perspective historiographique pour mieux cerner l'état des recherches qui ont été conduites sur le monachisme de cette période, avant d'appréhender la question des origines et du développement de la structuration des monastères en réseaux de dépendances.

Les grandes tendances actuelles de l'histoire monastique ne peuvent se comprendre en dehors des écoles historiques et idéologiques dans lesquelles elles s'inscrivent et dont elles sont les héritières, d'où la nécessité de proposer un bilan des problématiques et des apports de chacun. On peut schématiquement distinguer deux principales approches. La première, qui est aussi la plus ancienne, se coule dans le moule traditionnel des catégories historiques qui tronçonnent les thématiques de recherche en espaces bien délimités, voire imperméables : le politique, l'économique, le culturel, l'idéologique ou les mentalités... Dans cette logique, le monachisme est appréhendé comme un aspect spécifique de l'histoire religieuse, dont on cherche surtout à comprendre les aspects structurels et fonctionnels à travers des études de cas souvent monographiques. Plus récemment, sous l'influence des écoles anglo-saxonnes et des apports de l'anthropologie à l'histoire, le monachisme et les monastères ont été appréhendés sous l'angle plus large de l'histoire sociale, comme des éléments structurant la société chrétienne, d'une part dans une dialectique spirituelle entre l'icibas et l'au-delà, mais aussi comme des enjeux au sein de la hiérarchie des pouvoirs. 
11 À la notable exception du colloque sur la " Naissance et le fonctionnement des réseaux monastiques et canoniaux» (1991), la problématique de l'essor des réseaux monastiques n'a ainsi été étudiée qu'à travers quelques cas bien documentés d'abbayes qui furent aussi des foyers de réforme, comme Gorze, Fleury, Brogne et surtout Cluny. Le poids de la réussite clunisienne, Ecclesia puis ordo, et l'impact majeur du modèle que véhicule l'établissement mâconnais, bien connu par les nombreuses études sur le réseau monastique qu'il a généré, ne saurait pour autant masquer les disparités qui coexistent entre les formes de vie régulière. Comme dans le monde germanique, où le monachisme de type carolingien, soumis à l'autorité de l'ordinaire, est resté la norme, de nombreux monastères de Francie occidentale ont profité de la réforme pour accroître leurs possessions et le nombre de leurs dépendances, sans pour autant parvenir à une structuration en Ecclesia de type clunisien. La recherche a, jusqu'à présent, largement délaissé ces établissements. En prenant appui sur plusieurs exemples particuliers - Marmoutier, Tournus, Saint-Germain d'Auxerre... -, N. DeflouLeca a cherché, d'une part, à examiner l'impact de l'élan réformateur sur le développement de ces seigneuries monastiques, puis sur leur structuration en réseaux de dépendances ou en congrégations et, d'autre part, à comprendre la nature des liens qui unissent les membres d'un même réseau.

\section{Évêques, chanoines et moines, une pratique sociale différentiée}

Le second volet des communications entendait ouvrir la voie du comparatisme au sein des formes de vie existant au sein de l'Église, mais aussi sur le plan géographique, de façon à mettre en lumière l'originalité du premier Cluny, et plus largement du mouvement réformateur bourguignon, en regard des pratiques de restauration monastique développées par Gérard de Brogne, spécialement en direction des communautés canoniales.

Charles Mériaux, «Moines, chanoines et réformes en Flandre ( $\mathrm{x}^{\mathrm{e}}-\mathrm{XI}{ }^{\mathrm{e}}$ siècles)», s'est penché sur le cas de la Flandre, qui présente une situation paradoxale si l'on cherche à distinguer les caractères de la réforme du monachisme et la redéfinition de ses rapports avec la société dans l'Empire et le royaume de France au $\mathrm{x}^{\mathrm{e}}$ et au début du $\mathrm{XI}^{\mathrm{e}}$ siècle. Prince «français », le comte Arnoul I ${ }^{\mathrm{er}}$ (918-965) est en effet celui qui noua les rapports les plus étroits avec le réformateur lotharingien Gérard de Brogne. Il en alla de même avec certains de ses successeurs, notamment le comte Baudouin IV et Richard de Saint-Vanne. Ces protagonistes et leurs œuvres sont bien connus grâce aux travaux de Dom Dauphin, Dom Misonne et A. Dierkens, récemment remis en perspective par B. Meijns et S. Vanderputten. En prolongement de ces travaux, la communication a cherché à étudier la place respective occupée par les communautés monastiques et canoniales dans le cadre des vagues de réformes des $\mathrm{x}^{\mathrm{e}}$-XI $\mathrm{e}^{\mathrm{e}}$ siècles, étant entendu que, pour une bonne partie d'entre elles, les communautés de chanoines n'ont perdu ni leur statut ni la faveur des pouvoirs.

Dans un premier temps, C. Mériaux est revenu rapidement sur l'insertion, dans la société et l'aristocratie locales, de ces deux modèles de vie religieuse - la prière et le service pastoral -, et sur les rapports différents établis avec les autorités laïques - le comte des Flandres et ses châtelains -, aussi bien qu'avec l'épiscopat de la région. B. Meijns a récemment précisé les intuitions de J. Dhondt concernant une réelle 
politique canoniale des comtes de Flandre, au moment même où ils encourageaient la réforme monastique.

Deuxième question abordée : l'insertion différenciée des deux types de communautés dans l'environnement économique au sens large. Dans la dynamique des travaux d'H. Noizet sur Tours, C. Mériaux a cherché à examiner les conséquences spatiales de la présence de communautés monastiques et/ou canoniales, mettant ainsi en évidence les potentialités plus riches offertes par les communautés canoniales, qui peuvent, en partie, expliquer l'essor urbain en Flandre dès le $\mathrm{x}^{\mathrm{e}}$ siècle.

Pour finir, il a souligné les aspects complexes de la réalité flamande. Monde monastique et monde canonial ne vécurent en rien des destins séparés, mais connurent à certains endroits une forme de symbiose. Outre les passages de l'un à l'autre ordre, il convient de rappeler le cas des chapitres dépendant d'abbayes bénédictines, tels ceux qu'a naguère étudiés Dom Misonne pour le diocèse de Liège.

\section{"Culte » et " culture » : le monachisme réformateur tardo- carolingien et pré-grégorien}

17 A-t-on déjà oublié l'époque pas si lointaine (les années 1950) où Dom K. Hallinger distinguait, avec une rigueur qui paraît aujourd'hui outrancière, un monachisme de "culte» et un monachisme de "culture", propres à différentier les deux modèles réformateurs de "Kluny» (avec un « $K » !$ ) et de Gorze, l'un emblématique d'une liturgie débordante appelée à se perdre dans le faste seigneurial, l'autre tenant d'une culture dans la tradition des productions scripturaires du monachisme carolingien, relayé par celles des grands centres monastiques impériaux de l'époque ottonienne? Les travaux des vingt-cinq dernières années sont venus remettre en cause la légitimité de cette construction historiographique, d'une part, pour mettre au contraire l'accent sur l'importance des productions écrites et artistiques du premier Cluny - spécialement sous les gouvernements d'Odon, de Maïeul et d'Odilon -, d'autre part, pour insister sur la logique et les implications sociales de la «scripturalité » des moines grands seigneurs que deviennent rapidement les Clunisiens, promoteurs et propagandistes d'une société d'ordres emportée par l'élan hiérarchique impulsé par une Église de purs, c'est-à-dire de moines.

On aurait aimé revenir sur les implications ecclésiologiques de la "culture» clunisienne, auquel Odon donne une impulsion décisive si bien étudiée par I. Rosé ; on aurait aussi voulu, à la suite des travaux de J. Vezin, H. Atsma et S. Barré, offrir une vision d'ensemble des productions écrites clunisiennes pour rendre manifeste l'articulation essentielle entre vision idéale du monde - sur le versant ecclésiologique et pratiques de terrain des grands seigneurs clunisiens, qui disent tant d'eux-mêmes dans leurs chartes et leurs cartulaires. Faute de temps, dans le cadre d'une rencontre qui suppose de faire des choix, on y a renoncé pour privilégier les recherches les plus récentes sur la liturgie, mais d'une liturgie envisagée comme mode d'expression cohérente avec l'ensemble des mises en forme de l'idéal monastique proposé par la première « culture » clunisienne : hagiographie, historiographie, diplomatique...

19 Julian Hendrix, «La liturgie dans le monachisme carolingien: historiographie des manuscrits de Saint-Gall », a proposé de remonter à l'archétype monastique carolingien pour comprendre l'importance culturelle, politique et sociale de la prière d'intercession que cluny portera à une échelle inégalée. Les $\mathrm{VIII}^{\mathrm{e}}$ et $\mathrm{IX}^{\mathrm{e}}$ siècles 
représentent une période particulièrement riche dans le développement de la liturgie latine, les monastères étant des lieux particulièrement féconds et novateurs en la matière. Un panorama des recherches récemment menées sur les manuscrits liturgiques de Saint-Gall a permis de faire une mise au point d'ensemble sur la liturgie monastique carolingienne. Saint-Gall est le type même du monastère carolingien, qui a transmis à la postérité une quantité anormalement importante de livres liturgiques propres à mener une étude de cas, dont il est possible de tirer des enseignements d'une portée dépassant ce seul monastère. Cette synthèse a préparé le terrain à une discussion des théories de K. Hallinger sur les rapports entre les pratiques liturgiques carolingiennes et clunisiennes.

C'est également une perspective historiographique qu'a adoptée Susan Boynton à l'étude de « La liturgie de Cluny avant l'abbatiat d'Hugues de Semur ». Pour des raisons diverses, il est difficile de comprendre les pratiques liturgiques clunisiennes antérieures aux années 1050. Tout d'abord, il est essentiel de distinguer entre les sources de Cluny même et nombre de textes utilisés pour forger et généraliser l'idée d'une pratique liturgique "clunisienne", attestée en fait dans des lieux et à des époques variés. Beaucoup de sources dites «clunisiennes" n'ont ainsi qu'un rapport ténu avec l'abbaye de Cluny. En réalité, les sources liturgiques clunisiennes stricto sensu antérieures à l'abbatiat d'Hugues de Semur (1049-1109) sont très rares. Deux manuscrits contiennent des parties du lectionnaire de matines: PARIS, BnF, nal 2390, de la première moitié du XI $\mathrm{e}^{\mathrm{e}}$ siècle - de la Pentecôte à octobre - ; PARIS, BnF, lat. 13371, fol. 87-96, du $\mathrm{x}^{\mathrm{e}}$ siècle - liste des lectures censée refléter les pratiques liturgiques contemporaines d'Odon. Les coutumiers $\mathrm{du} \mathrm{xl}^{\mathrm{e}}$ siècle n'apportent qu'un témoignage indirect, dans la mesure où l'on ne possède aucune rédaction provenant de cluny même. Il s'agit des Consuetudines antiquiores, un ensemble hétéroclite qui regroupe toute une constellation de textes, qui entretiennent avec Cluny des rapports variables, et du Liber tramitis - CITTÀ DEL VATICANO, Vat. lat. 6808 -, copié à l'abbaye de Farfa vers 1050, dont le texte semble avoir été composé sur la base d'informations recueillies à Cluny sous l'abbatiat d'Odilon (994-1049). Des textes hagiographiques, telle la Vie d'Odon par Jean de Salerne, font référence aux pratiques liturgiques, mais on doit y recourir avec prudence et un grand esprit critique. C'est sur ces bases documentaires tardives et largement extérieures, que K. Hallinger a développé ses théories sur la liturgie du premier Cluny, lesquelles ont été rediscutées sur la base des recherches les plus récentes.

21 Après cette double approche historiographique de la liturgie clunisienne, Eduardo Henrik Aubert a abordé la question de l'écriture du chant à partir d'une étude de cas, le Graduel de Cluny conservé à la Bibliothèque nationale de France - PARIS, BnF, lat. 1087, seconde moitié du XI ${ }^{e}$ siècle -, seul témoin des chants de la messe qu'on peut attribuer au scriptorium de Cluny. Il s'agit d'un manuscrit complexe, pour lequel une analyse codicologique et paléographique est encore à faire. Cette communication a permis d'offrir les principaux résultats d'une telle analyse, en s'occupant surtout du problème de l'écriture neumatique. Ce manuscrit a été longtemps en usage ; les ajouts successifs qui s'y sont superposés donnent donc une idée de l'évolution de la pratique de l'écriture du chant à Cluny. Après ces réflexions liminaires, l'exposé s'est développé en quatre étapes :

- présentation de la structure codicologique du manuscrit, avec un essai d'identification des feuillets manquants et de ceux qui ont été ultérieurement ajoutés ; 
- analyse paléographique du texte et de la musique, afin d'établir le nombre de scribes qui se sont occupés du manuscrit, avec une distinction entre les mains qui sont intervenues dans la première campagne de fabrication du manuscrit et celles qui se sont ajoutées postérieurement ;

- analyse des grattages et corrections dans l'écriture neumatique effectuées par le notateur principal (environ 120 corrections) ;

- distinction des logiques de l'écriture du chant telle qu'elle était pratiquée par chacun des notateurs, ce qui a mis en relief les continuités dans la pratique d'écriture musicale dans le scriptorium et ses transformations fondamentales au fil du temps.

\section{Des mondes du visuel} "artistiques", à la suite notamment des travaux de H.L.Kessler, il convient d'envisager de façon différente les rapports des moines aux réalisations de l'art et aux mondes du visuel, tous supports confondus. En effet, du $\mathrm{IX}^{\mathrm{e}}$ au $\mathrm{XI}^{\mathrm{e}}$ siècle, dans une conjoncture tout empreinte de réformes et de reformulations esthétiques, dans les textes comme dans les images, sans cesse en lien avec les nécessaires actualisations de la liturgie, un ensemble de mouvements de fond aboutit au dépassement du sens iconographique et à la réévaluation de l'art et de sa matérialité, ars et materia, libérant $\mathrm{du}$ coup un prodigieux essor du visuel.

Russo et Pierre-Alain Mariaux l'ont étudié, d'abord, à travers la mise en forme, à partir des textes, mais parfois aussi loin d'eux, d'une "esthétique de la présentation ", qui insiste à la fois sur les matériaux et les effets obtenus à partir d'eux - y compris les effets de couleurs -, sur le caractère «inaugural» de chacune des réalisations entreprises, quel que soit le support choisi, sur l'autonomie acquise, enfin, par rapport au dogme de l'Église, chaque œuvre, opus, donc travail accompli, s'affirmant selon ses lois propres et son organisation particulière par rapport aux autres "choses" du monde extérieur. C'est alors que la notion de faire et de savoir-faire s'impose dans toutes ses dimensions, avec ses critères et son originalité, de manière décisive au sein d'un réseau complexe d'agents - monastères, clients et protecteurs, abbés réformateurs et moines théologiens, commanditaires et destinataires -, de choses produites et mises en circulation, sans cesse à l'articulation entre l'extérieur et l'intérieur des communautés.

En privilégiant deux formes de supports d'art, travaillés et enrichis dans ces communautés monastiques, le parchemin et le mur, et autour de la pensée et de l'organisation des décors, sur les feuillets des livres manuscrits au sein du premier scriptorium de Cluny, sur les peintures murales dans les monastères et sur les mosaïques autour des absides des basiliques, de part et d'autre des Alpes, dans des aires régionales distinctes mais complémentaires, Fabrizio Crivello et Barbara Franzé ont présenté des études de cas, détaillés et documentés, autour de ces installations et de ces commandes monastiques, en insistant sur la part croissante du visuel dans tout le processus en cours.

Lors du débat, Pierre-Alain Mariaux, Didier Méhu et Daniel Russo, les intervenants et les participants, sont revenus sur quelques faits marquants: chronologie de ces réalisations, souvent de prestige, entre $\mathrm{IX}^{\mathrm{e}}$ et $\mathrm{XI}^{\mathrm{e}}$ siècle; relations, plus ou moins fondées, avec les projets des réformes monastiques en cours; développements d'une 
conception nouvelle des arts et, surtout, du visuel, dans ces ambiances relevant à la fois de l'ordre de la pensée, du métier artistique, du religieux ; nécessaires élargissements à d'autres modes de compréhension et d'expression, vers la même époque, dans les champs du textuel et de la "figuration ", à la rencontre de plusieurs traditions, au croisement aussi de différentes mémoires du social.

\section{L'espace monumental des moines réformateurs}

La période de fondation de Cluny nous invite à réfléchir sur l'application réelle ou supposée, dans l'espace, des grandes réformes monastiques carolingiennes. La rencontre de Romainmôtier a fourni l'occasion de faire point sur cette question entre le concile d'Aix-la-Chapelle de 817 et 910 , en prolongeant notre regard jusqu'à la fin du $\mathrm{x}^{\mathrm{e}}$ siècle et au début du $\mathrm{XI}^{\mathrm{e}}$ siècle. Qu'est-ce qui change? Comment les nouvelles fondations intègrent-elles ce retour à la règle de vie commune? Comment cela se traduit-il dans l'espace claustral ? Comment le monastère est-il désormais pensé ? Si on s'est intéressé, ces dernières années, à travers l'archéologie notamment, aux prémices de l'organisation spatiale des premiers monastères - de Lérins aux fondations colombaniennes -, on peine encore à saisir les mutations et les constitutions sur le terrain d'une organisation monastique qui semble immuable après le $\mathrm{XI}^{\mathrm{e}}$ siècle. Ainsi, le plan idéal de Saint-Gall que l'on croit voir se réaliser, ici et là jusqu'au Liber tramitis, dans l'organisation de l'espace, reflète-t-il une réalité perceptible en différents points d'Europe ? Sans pouvoir, faute de temps, couvrir tous les pays, il a été fait appel à deux chercheurs pour aborder les cas de l'Italie et de l'Espagne: Eleonora Destefanis et Immaculada Lorés. En se référant aux fouilles qu'il a menées à Saint-Gall, Hans Rudolf Sennhauser a prolongé ces réflexions sur les implantations monastiques et religieuses à la fin du haut Moyen Âge. Enfin, Anne Baud et Christian Sapin ont rendu compte des hypothèses propres aux bâtiments monastiques de Cluny, sur la base des toutes dernières campagnes archéologiques.

\section{Second volet : Cluny, 9-11 septembre 2010}

\section{Leçons inaugurales}

Le second volet du colloque était intitulé : «Cluny, le monachisme et l'émergence d'une société seigneuriale ». Trois leçons inaugurales ont permis d'aborder le problème des origines de Cluny replacées dans les années de genèse du "premier âge féodal ». La communication d'Isabelle Rosé, "Les origines de Cluny, le Cluny des origines", entendait faire le point sur les premiers temps du monastère de Cluny, en revenant sur ce que signifie la fondation de l'abbaye dans son contexte historique et social en mettant en perspective les premiers temps de Cluny, sur les plans tout à la fois matériel, social et idéologique, à une période de bouleversements sociaux et politiques importants qui touchent le monde aristocratique et qui se caractérisent par un effacement progressif des structures carolingiennes et par la genèse des cadres d'une société seigneuriale. Dans cette perspective, le Cluny des origines, en tant que microcosme peuplé d'aristocrates extrêmement bien insérés dans la noblesse du siècle et qui sont, de surcroît, des hommes de plume, apparaît comme un révélateur de cette période de transition qui voit l'émergence de nouvelles formes sociales. 

changements en cours, fin $\mathrm{IX}^{\mathrm{e}}$-début $\mathrm{x}^{\mathrm{e}}$ siècle, en insistant sur un aspect particulier, largement négligé dans les travaux sur les origines de Cluny : le rôle des femmes, en partant d'Engelberge, fille de Boson et d'Ermengarde, sœur du roi Louis l'Aveugle, belle-sœur du roi Rodolphe de Bourgogne, nièce du duc de Bourgogne, qui finit ses jours à Saint-Sixte de Plaisance. Après cette étude serrée du réseau aristocratique et princier à la genèse de Cluny et du monachisme réformateur tardo-carolingien, Steffen Patzold s'est interrogé sur la pertinence de la séquence chronologique choisie pour traiter des changements sociopolitiques contemporains de la fondation de Cluny. Quel profit peut-il y avoir à dépasser l'horizon de la "Révolution de l'an Mil », chère à l'historiographie française d'après-guerre, pour faire retour au premier âge féodal défini par Marc Bloch (880-1050) ? Ce débat historiographique à l'arrière-plan de l'ensemble de la rencontre a été abordé du point de vue de l'histoire de l'Empire, jamais affectée par la césure de l'an Mil.

\section{Perspectives générales}

La première section, « Les moines, la seigneurie, l'encadrement des hommes au premier âge féodal» a permis d'offrir des perspectives générales, ensuite confrontées à des études de cas régionales.

Les perspectives générales sur le problème de la seigneurie ont été fournies de deux points de vue complémentaires sur les transferts de propriété constitutifs de la seigneurie et des structures seigneuriales constituées par les communautés monastiques.

31 Eliana Magnani, « Les moines et les circuits de l'“échange” ", a proposé, en lieu et place du terme "échange », dont l'emploi de plus en plus générique et enveloppant tend plutôt à voiler qu'à éclairer la compréhension des rapports sociaux, de traiter des "transferts", tels qu'ils sont désignés dans les actes diplomatiques bourguignons (surtout clunisiens) dans un large $\mathrm{x}^{\mathrm{e}}$ siècle, réunis dans la base des Chartae Burgundiae Medii Aevi et interrogés avec le logiciel Philologic. En prenant comme point d'observation les formulaires et les formules énumératives de différents types de transfert et leurs transformations, on constate d'abord une assez longue continuité par rapport à l'époque carolingienne. Mais les formulaires sont aussi ponctués de changements, qui concernent, entre autres, aussi bien les relations de synonymie/ antonymie que l'introduction de la langue vernaculaire, le sens de ces variations devant être mis en perspective avec l'abandon de ces formules entre la fin du $\mathrm{x}^{\mathrm{e}}$ et le milieu du $\mathrm{XI}^{\mathrm{e}}$ siècle, au profit d'une "singularisation", voire d'une réduction, des syntagmes énonçant les transferts. Eu égard à l'historiographie inspirée par la théorie maussienne, elle-même imprégnée par les études juridiques et linguistiques germanistes du XIX ${ }^{e}$ siècle, qui depuis les années 1970/1980 environ caractérise l'« économie » du haut Moyen Âge comme une «économie du don" - par opposition à une "économie de marché »-, ces observations sur la mise en registre des transferts par les moines invitent à ne pas confondre le type de transfert avec les discours sur les transferts véhiculés par les actes, et à revisiter ainsi l'« économie » du premier âge féodal à l'aune d'une pratique finalement encore assez mal connue de l'écrit.

32 En l'absence de Jean-Pierre Devroey, excusé, mais sur la base du dossier fourni par l'auteur, Michel Lauwers, a tenté de mesurer l'écart existant entre le grand domaine 
carolingien et la seigneurie monastique de type clunisien. Sous l'intitulé général, « Les moines grands seigneurs entre incastellamento et inecclesiamento ", il s'est efforcé de cerner les logiques spatiales du féodalisme, de l'incastellamento à l'inecclesiamento, en partant de la thèse de Pierre Toubert, avec la formulation du concept d'incastellamento dans les années 1970, la généralisation du modèle par Robert Fossier avec l'« encellulement » et la naissance du "village », puis les correctifs apportés par les archéologues - R. Francovich, É. Hubert, etc. - obligeant à envisager ces transformations dans la longue durée, à décomposer les différents éléments du phénomène incastellamento, mais aussi à mettre en valeur le rôle des lieux de culte dans l'organisation spatiale et sociale, avec un «tournant » aux VII et vIII siècles.

Le point a ensuite été fait sur les recherches relatives aux représentations de l'espace, avec la formulation de nouveaux paradigmes (A. Guerreau): des lieux qui "polarisent", ce qui peut favoriser, mais ne renvoie pas systématiquement à un regroupement au sens topographique du terme, les phénomènes de polarisation pouvant être très progressifs (É. Zadora-Rio), avec un rôle structurant de l'église dans le cas des habitats dispersés. M. Lauwers a souligné à quel point la réflexion suppose d'être sensible à la diversité d'échelles et à la différence des niveaux de réalité qu'il convient de traiter à l'aide d'un vocabulaire diversifié: polarisation, topographie, morphologie. En dépit de la complexité des phénomènes étudiés, on peut parler d'un processus continu de polarisation ecclésiale, avec concentration des sépultures autour des lieux de culte, qui s'accompagne d'une indistinction croissante des tombes réductions, recoupements, enchevêtrements; regroupements par sexes et classes d'âge - ; dans un certain nombre de cas, on a affaire à de nouvelles formes d'articulation avec l'habitat. La chronologie du phénomène suppose de distinguer les étapes suivantes :

- le moment carolingien, dans un contexte d'institutionnalisation de l'Église au cœur des réflexions et des débats des évêques : multiplication et/ou stabilisation des lieux de culte ; directives relatives à leur fréquentation ; prise en considération de la distance séparant l'habitat et le lieu de culte ; premières définitions de la " paroisse » et généralisation de la dîme ; prise en charge des églises patrimoniales par l'institution ecclésiastique ;

- la mise en forme liturgique de ce tournant institutionnel, au $x^{\mathrm{e}}$ et dans la première moitié du $\mathrm{XI}^{\mathrm{e}}$ siècle : les ordines de consécration d'église et de cimetière ; le circuitus et l'extension du sacré ; la définition rituelle d'espaces protégés et d'espaces sacrés (sacraria, etc.) ; le cas particulier des « villages ecclésiaux »;

- la documentation écrite atteste un renforcement de la polarisation ecclésiale au XI ${ }^{\mathrm{e}}$ siècle : le locus ecclésial s'impose comme point de repère dans les actes de la pratique, avec un recours de plus en plus fréquent à l'entité de la parrochia ; les chartes de fondation et de consécration, tout comme les sermons de consécration, permettent une nette mise en valeur du monument ecclésial ;

- au terme de cette évolution, le mouvement grégorien est porteur d'une idéologie de la séparation ; mais qu'en est-il de la mise en œuvre de ce programme?

Ce sont toutes ces données, convergentes, renvoyant à une organisation sociale et à une représentation de l'espace polarisé autour de l'église et de son environnement, que M. Lauwers a proposé de désigner par le terme d'inecclesiamento. L'inecclesiamento désigne la mise en place et la multiplication des édifices ecclésiaux, la sacralisation et la structuration, selon un processus lent mais continu, de formes de vie sociale autour des lieux de culte et de leurs zones funéraires, ainsi qu'une nouvelle représentation de l'Église. Tandis que les premiers chrétiens avaient fortement proclamé le caractère 
immatériel de l'Église, l'institution ecclésiale du Moyen Âge fut créatrice d'espace social. L'enracinement de l'Église dans un lieu entraîne en effet une hiérarchisation de l'espace, induite par le rituel de consécration de l'église et du cimetière, et déterminée par la distance séparant tel ou tel point de l'autel, considéré comme le «saint des saints".

Ainsi défini, le processus d'inecclesiamento, qui caractérisa l'Occident à partir du haut Moyen Âge, ne renvoie pas seulement (ni même prioritairement) à un phénomène de concentration des populations autour de points fixes, à la différence des réalités que supposent les concepts d'incastellamento, encellulement ou ensagrarement, qui désignent tous des moments (généralement de faible durée) de recomposition matérielle de l'habitat consistant en un rassemblement de la population autour d'un château ou d'une église. La réorganisation de l'habitat autour - mais aussi, plus largement, en fonction des églises et de leurs sépultures - n'est qu'une manifestation parmi d'autres d'un processus social pluriséculaire d'ancrage et d'extension du sacré, qu'il faut envisager, tout à la fois, comme un mouvement d'inscription de l'Ecclesia au sein de la société et comme l'inclusion de l'ensemble des fidèles au sein de l'Ecclesia. Contrairement à l'incastellamento et à ses dérivés, ce processus comporte une importante part idéelle: il s'accompagna, du reste, d'une abondante production idéologique émanant des clercs, qui se traduisit donc par des rites et des discours. Le tropisme exercé par les lieux sacrés et les formes de territorialisation, que, très progressivement, ils suscitèrent, reposaient sur l'image d'une Ecclesia faite de tous les fidèles, vivants et défunts, identifiée au corps social et ancrée localement.

M. Lauwers s'est enfin attaché à apprécier la place des moines et des monastères dans le processus d'inecclesiamento, ce qui supposait à la fois de s'interroger sur l'espace monastique au cours du haut Moyen Âge - le monastère comme lieu de cohabitation des vivants et des morts; le monastère comme espace fonctionnel structuré avec ses différents bâtiments et ses circulations comme modèle social - et de faire retour à la question de l'incastellamento vs. inecclesiamento, qu'il ne convient pas de poser sous forme d'opposition, l'ecclesia monastique pouvant parfaitement être assimilée à un castrum, comme c'est le cas à Cluny ou à Fleury. Sans oublier que l'étude du rôle des monastères dans l'incastellamento, comme l'a noté P. Toubert, place l'historien face à une "révélation documentaire » - données provenant de cartulaires d'établissements religieux - et à une logique renvoyant à la rationalité monastique: la congregatio hominum de la curtis à l'incastellamento. C'est à ce propos que l'étude du passage du grand domaine carolingien à la seigneurie monastique peut se révéler cruciale. Pour finir, M. Lauwers s'est attardé sur la place du monachisme comme institution d'espaces "sacrés " séparés, grâce à l'immunité, l'exemption, et la définition de circuits de possessions monastiques dans le haut Moyen Âge, puis avec la sacralisation des terres monastiques à l'époque de la réforme grégorienne - Léon IX, à Romainmôtier; Urbain II, à Cluny, et la délimitation du «ban sacré ».

\section{Les études de cas régionaux}

37 Ont ensuite été proposées quatre études de cas régionaux. 
Florian Mazel, «La dualité des centres de pouvoir : pôles castraux, communautés canoniales et communautés monastiques dans l'ancienne province de Tours ( $\mathrm{x}^{\mathrm{e}}$ $\mathrm{XI}^{\mathrm{e}}$ siècles)»

La principale évolution des lieux de pouvoir aristocratiques aux $\mathrm{x}^{\mathrm{e}}$-XI $\mathrm{x}^{\mathrm{e}}$ siècles tient au recouvrement des fonctions résidentielle et militaire, largement dissociées durant l'époque carolingienne. Il convient cependant de mettre l'accent sur une autre évolution, tout aussi remarquable. Si les chapelles des palais royaux carolingiens étaient déjà desservies par des communautés canoniales, dans l'Ouest de la Francie, les palais du $\mathrm{IX}^{\mathrm{e}}$ siècle ou de la première moitié du $\mathrm{x}^{\mathrm{e}}$ siècle (Doué, Mayenne, Andonne) ne paraissent pas avoir été dotés, à l'origine, d'un pôle ecclésial notable, encore moins d'un chapitre canonial. En revanche, les nouveaux pôles castraux qui se multiplient après les années 950-960 sont très souvent pourvus de petites communautés ou de groupes de clercs généralement désignés comme chanoines. Ce phénomène, qui commence dans certaines régions dès le tournant des $\mathrm{IX}^{\mathrm{e}}-\mathrm{X}^{\mathrm{e}}$ siècles et peut se prolonger ailleurs dans un long $\mathrm{xl}^{\mathrm{e}}$ siècle, illustre le caractère biface, laïque et ecclésiastique, de la domination aristocratique locale au premier âge féodal, jusqu'en son cœur: la forteresse-résidence seigneuriale, tout en témoignant de la diffusion d'un modèle royal (la capella) et urbain (la civitas) médiatisé par les princes. Les églises castrales, ainsi desservies avec plus de lustre que les autres églises rurales et souvent pourvues de droits paroissiaux dérogatoires limitées à la population castrale, renforcent « l'exceptionnalité » du lieu et de ses habitants. La constitution comme l'exercice de la seigneurie canoniale viennent surtout renforcer les liens tissés avec les fidèles et vassaux, d'autant que les chanoines semblent issus des familles de milites castri et demeurent, eux et leurs biens, sous l'étroit contrôle du maître du château. À partir du début $\mathrm{du} \mathrm{XI}{ }^{\mathrm{e}}$ siècle, le recours de plus en plus fréquent aux moines - ex nihilo ou par substitution à d'anciens chanoines - ne transforme pas radicalement la situation, même s'il reflète l'élargissement des réseaux aristocratiques - l'appel à des moines venus de grands établissements implique une sortie du local - et l'essor de logiques propres aux communautés monastiques - la structuration d'un réseau de dépendances, une plus grande autonomie seigneuriale -, et débouche, par conséquent, sur des configurations sociales et politiques plus complexes.

\section{Laurent Ripart, «Vie religieuse et société dans la moyenne vallée du Rhône,} 950-1050»

L'auteur a traité de l'espace régional rhodanien, autrement dit des espaces centraux du royaume de Bourgogne, à l'exclusion des marches princières des comtés de Provence et de Bourgogne, mais aussi des espaces frontaliers de l'Argovie et de l'Ouest lyonnais - en particulier la seigneurie de Savigny. Dans ce cadre, il s'est interrogé sur trois points : Quels peuvent être les caractères originaux du premier âge féodal dans le monde rhodanien? Quels sont dans cet espace les grands traits de l'organisation de la vie religieuse ? Le premier âge féodal peut-il être considéré comme un bloc?

À la première question, la réponse est simple : ce qui caractérise le premier âge féodal dans le monde rhodanien, c'est l'organisation de la société autour de la royauté. En milieu rhodanien, la royauté émerge dans les années 850-870 - Charles de Provence puis Boson -, triomphe à la fin du IX $\mathrm{I}^{\mathrm{e}}$ siècle, avec les couronnements de Rodolphe $\mathrm{I}^{\mathrm{er}}$ et de Louis, pour disparaittre définitivement autour du milieu du $\mathrm{XI}^{\mathrm{e}}$ siècle - mort de 
Rodolphe III, en 1032, et d'Henri III, dernier roi de Bourgogne, en 1056. Durant ces presque deux siècles, l'institution royale a organisé l'ensemble de la société, la cour du roi étant le lieu d'exercice et de partage du pouvoir, mais aussi de distribution des richesses et des pouvoirs. Elle a ainsi défini le faciès des élites régionales, qui s'organisent sous la forme d'une société curiale, attachée à la recherche de la proximité royale.

41 À la deuxième question, la réponse est aussi simple : si les rois de Bourgogne ont été des amis de Cluny, ils n'ont pas manifesté beaucoup d'empressement pour importer sur leurs terres les principes qui organisaient la vie religieuse clunisienne. Dans l'espace rhodanien, le premier âge féodal correspond globalement à une période d'affaiblissement de la vie religieuse : beaucoup d'établissements disparaissent, plus ou moins totalement, alors que d'autres sont transformés en chapitres canoniaux. Par ailleurs, ces établissements disposent d'une autonomie très limitée, puisqu'ils sont soit dépendants de l'autorité royale - au $\mathrm{x}^{\mathrm{e}}$ siècle, le roi est par exemple abbé laïc de SaintMaurice d'Agaune, de Saint-André-le-Bas de Vienne et de Romainmôtier -, soit placés sous la juridiction de l'ordinaire. Ces conditions pèsent sur les relations entre ces établissements et la société environnante: si les élites locales se font volontiers enterrer dans les monastères, y placent leur progéniture et souhaitent s'associer par des dons au bénéfice de la prière monastique, leurs relations avec les moines sont nettement en retrait de celles que «les voisins de Saint-Pierre » peuvent entretenir avec Cluny.

42 À la troisième question, la réponse est plutôt non, car il semble que les alentours de l'an Mil constituent, dans l'espace rhodanien, une césure non négligeable pour plusieurs raisons. Tout d'abord, parce que l'an Mil correspond à une vague réformatrice qui modifie profondément l'organisation de la vie religieuse, ce qui change quelque peu la donne. D'autre part, parce que dans toute la moitié sud de cet espace régional, le pouvoir royal disparaît totalement entre 1010 et 1020, pour être remplacé par des principautés ecclésiastiques ou/puis laïques, ce qui transforme en profondeur le profil de la formation sociale. Enfin, parce que se met en place un très dense réseau de prieurés, qui témoignent de la réorganisation de la société autour de nouveaux lieux de pouvoirs locaux, en rupture avec l'ancienne organisation curiale de la société régionale.

En conclusion, on peut donc dire que, vu de l'espace rhodanien, "le premier âge féodal » a bien existé, même s'il est nécessaire de souligner, que, durant la première moitié $\mathrm{du} \mathrm{XI}^{\mathrm{e}}$ siècle, de très profondes évolutions modifient substantiellement la formation sociale. Pour autant, on peut toutefois considérer qu'entre Boson et Henri III, il a bien existé dans cette région un même modèle de société féodale, avec des traits structurants bien précis, qui forme ce que l'on pourrait définir comme un modèle curial de société féodale, autrement dit une société où les pouvoirs féodaux s'organisent autour de la cour royale, ce qui n'est certainement pas le type de société le plus propice pour l'éclosion d'un modèle monastique de type clunisien.

\section{Laurent Schneider, « Autour de Saint-Sauveur d'Aniane : la territorialisation des pouvoirs et l'encadrement de l'habitat à l'échelle locale »}

On a pu dire que Benoît d'Aniane avait préparé l'œuvre de Cluny, mais si l'on a beaucoup écrit sur Benoît lui-même, sur son œuvre ou sur les liens qu'il noua avec Guilhem de Gellone, le grand ancêtre de Guillaume le Pieux, l'histoire prégrégorienne des deux monastères septimaniens d'Aniane et de Gellone demeure passablement 
méconnue. Cette communication a proposé, à partir de l'histoire de leur patrimoine et d'une documentation archéologique désormais plus consistante, de mesurer comment ces deux établissements se sont progressivement inscrits dans l'espace rural local jusqu'au temps des premières dominations châtelaines. Trois périodes ont été prises en considération.

Le siècle des fondations entre la fin du viII ${ }^{e}$ siècle et celle du second tiers du IX ${ }^{e}$ est celui de l'implantation des deux monastères aux marges des territoires des cités de Nîmes, Maguelone, Béziers et Lodève, dans le contexte de l'intégration de l'ensemble géopolitique que constitue l'ancienne Septimanie wisigothique à l'Empire carolingien. Richement dotés par les souverains carolingiens de fiscs et de nombreux domaines, Aniane et sa fille Gellone apparaissent comme des instruments de "francisation " bénéficiant d'un rayonnement supra-régional. Les liens tissés entre les deux centres monastiques et leurs dépendances inaugurent, en Narbonnaise, une nouvelle constellation de relations entre des sites ruraux, entre des lieux et des hommes, entre des terres et des pouvoirs. Ces liens défient la géographie administrative héritée de l'Antiquité tardive, et surtout ils ne sont plus innervés par la cité ou les réseaux épiscopaux. Aux marges des cités, Aniane et Gellone contribuent dès lors à l'émergence d'une nouvelle forme de centralité, qui se construit à la périphérie des ressorts traditionnels du pouvoir.

La seconde période - fin du IX $\mathrm{X}^{\mathrm{e}}$-milieu du $\mathrm{x}^{\mathrm{e}}$ siècle -, la plus difficile à documenter car les cartulaires curieusement se taisent, est celle de la domination arlésienne. Dans un contexte géopolitique incertain, Aniane entre dans les mains des «Bourguignons » du royaume de Provence, Rostaing, puis surtout Manasses, archevêques d'Arles, probablement au moment où se constitue dans l'ancien pagus de Maguelone le nouveau comté de Substantion. Cette phase voit une première déconstruction des domaines et une séparation entre les deux monastères riverains du cours de l'Hérault, du fait de leur localisation sur des rives différentes et dans des ensembles territoriaux distincts.

La troisième période, celle de la seconde moitié du $\mathrm{x}^{\mathrm{e}}$ siècle, correspond au triomphe des pouvoirs vicomtaux, marqué par la reconstruction d'un temporel de proximité, et la course aux aumônes que se livrent les notables locaux. L'espace local est restructuré à la fois par l'institution de vicariae et par la construction précoce d'une première vague de fortifications : tours et castra. Deux personnages, l'évêque Fulcran de Lodève et l'abbé Rainard d'Aniane, hypothétiquement rattaché à la famille vicomtale de Béziers, dominent alors la scène locale jusqu'aux premières années du xie siècle. Symbole de la mutation qui s'opère, l'abbatiat d'Aniane passe ensuite à un personnage local, issu de la maison castrale de Gignac, l'une des forteresses implantées dans le plus proche voisinage du monastère.

\subsubsection{HélèneDebax, « Les comtes et le comté de Carcassonne de 900 à 1060 : expansion et disparition d'une grande principauté »}

L'auteur a examiné le cas d'une "région-test», le comté de Carcassonne, et de la dynastie de comtes qui l'a dirigé des années 930 aux années 1060 - une lignée issue de l'entourage de Guillaume le Pieux, qui prend pied en Carcassès dans le contexte des grands conflits autour du marquisat de Gothie et de l'archevêché de Narbonne au début $\mathrm{du} \mathrm{x}^{\mathrm{e}}$ siècle. L'enjeu a été de contribuer à une définition de la nature du pouvoir de ces 
comtes, princes " post-carolingiens » et " pré-féodaux », et de creuser la question de la spatialisation des dominations avant le milieu du XI ${ }^{\mathrm{e}}$ siècle.

Ces comtes héritent du pouvoir comtal sur deux espaces, les comtés de Carcassonne et de Razès, mais ils étendent rapidement leur domination dans des circonscriptions mineures (ministeria, pagi, vicariae), qui dépendent des comtés voisins de Toulouse et de Narbonne. Le vaste conglomérat de terres, léguées à ses héritiers par le comte Roger le Vieux vers 1002, s'étend en effet de la vallée de l'Ariège au Minervois. Grâce à de nombreuses sources, d'origine ecclésiastique mais aussi laïque, on peut faire le constat d'une disjonction au $\mathrm{x}^{\mathrm{e}}$ siècle entre une définition territoriale très claire des ressorts des comtés - essentiellement par le biais de la localisation des biens, objets de transactions - et un pouvoir comtal qui transcende ces limites.

Au-delà des sièges des comtés (Carcassonne et Rennes-le-Château), pour contrôler le territoire, le pouvoir comtal s'appuie sur un réseau de "hauts lieux ", associant bien souvent une abbaye et un château, relativement éloignés les uns des autres et commandant chacun une petite région. Là où ces lieux de pouvoir faisaient défaut, les comtes ont contribué à fonder ou refonder des abbayes (Saint-Hilaire, Lézat). L'osmose entre pouvoirs "laïques" et "ecclésiastiques" se révèle, comme partout, dans la maitrise des charges à la fois comtales, abbatiales et épiscopales, mais aussi dans l'extension de l'éphémère réseau monastique reconnu en 993 par le pape Jean XV au profit de l'abbé Garin : il épouse les limites des domaines de Roger le Vieux.

51 La circulation du pouvoir à l'intérieur de la famille comtale révèle une gestion collégiale, avec des frères ou des oncles et neveux codirigeant les comtés. Tous, et où que se situe le centre de gravité de leur domination, possèdent des droits sur Carcassonne. Mais la prolifération des ayants droit et une exacerbation des conflits internes entraînent une multiplication des litiges à partir des années 1020-1030, qui aboutit à la disparition de la dynastie comtale - sauf pour la branche à l'origine de la lignée des comtes de Foix.

Le pouvoir comtal entre le début du $\mathrm{x}^{\mathrm{e}}$ et le milieu du XI ${ }^{\mathrm{e}}$ siècle se définit donc sur des bases familiales plus que territoriales. Une délimitation spatiale des ressorts des circonscriptions a bien eu lieu - entre la fin du $\mathrm{x}^{\mathrm{e}}$ et le $\mathrm{x}^{\mathrm{e}}$ siècle -, mais elle n'est pas contraignante pour encadrer les dominations comtales. La seigneurie est absente : les transactions portent uniquement sur des alleux, sur des villae ou des parts de villae, et sur des églises et leurs droits attachés. Les sources n'attestent d'aucun fief, ni d'aucune taxe récognitive de seigneurie. Ce pouvoir comtal, plutôt que d'être dit "pré-féodal » ou « post-carolingien », parait pouvoir être qualifié de " princier ».

\subsection{Construire au $\mathrm{x}^{\mathrm{e}}$ siècle : démarche d'ensemble}

53 Cette seconde et dernière section avait pour objet d'apprécier l'ampleur et la portée sociale de l'acte de construire au tournant des années 900. On a d'abord cherché à justifier le terme "construire", qui semble pertinent de nos jours, alors que l'historiographie traditionnelle considérait qu'il n'était pas évident de construire dans cette période considérée comme troublée voire anarchique. Depuis quinze ans, l'archéologie de l'occupation du paysage, comme celle du bâti et de ses techniques, montre cependant qu'il n'existe pas réellement de vide et, que, si certaines traditions perdurent, les renouvellements se manifestent de façons multiples et variées selon les régions au cours $d u x^{e}$ siècle. Les nouvelles méthodes de datation par l'archéométrie 
permettent d'envisager l'existence de constructions que l'on n'osait pas dater avant l'an Mil, il y a encore dix ans. Ainsi, l'architecture castrale de pierre est désormais bien implantée et attestée - Ouest, Bourgogne, France méridionale -, et il n'y a pas lieu d'opposer architecture religieuse et castrale, du moins à partir des critères des matériaux et de mise en œuvre. Enfin, on ne peut attendre le fameux «blanc manteau de l'an Mil » pour voir l'Occident latin se couvrir de constructions. On commence ainsi à mieux cerner la présence de l'habitat, le choix des élites, les questions de défense et de regroupement, la formation des premières paroisses. En même temps que se redéfinit une réflexion globale sur un premier âge féodal, plusieurs archéologues - Luc Bourgeois, Anne Nyssen-Jaubert, Daniel Prigent, Anne Baud et Christian Sapin - ont apporté leur point de vue sur cette période de renouvellement du bâti comme manifestation du changement dans l'identification des lieux de pouvoir comme dans l'organisation du regroupement du religieux.

En conclusion, Pascual Martinez Sopena s'est demandé, au regard des contributions des deux jours, si l'on peut véritablement parler de "premier âge féodal monastique » et, dépassant le cadre général de la rencontre - la Francie occidentale, le Royaume de Bourgogne et la Gallia du Sud -, il a tenté de contraster la seigneurie clunisienne avec d'autres seigneuries ecclésiales et monastiques à l'échelle de l'Occident latin.

La journée du samedi 11 septembre a été consacrée, sous la forme d'une table ronde coordonnée par Anne Baud et Christian Sapin et d'une étude in situ,aux nouvelles données archéologiques mises au jour par les fouilles des années 2008-2009. Dans l'après-midi du samedi 11 et la matinée du dimanche 12 septembre, une série de conférences a permis de faire le point sur l'état général des études clunisiennes: Denyse Riche et Daniel-Odon Hurel, "Cluny, un ordre monastique à la fin du Moyen Âge et une congrégation parmi d'autres à la fin de l'Ancien Régime»; Franz Neiske, " Commémorer à Cluny. Les vivants et la mémoire des morts "; Neil Stratford, «Cluny et l'histoire de l'art »; Giles Constable, « Le futur des études clunisiennes ».

\section{INDEX}

Index géographique : France/Cluny

Mots-clés : colloque 